\title{
Causal Output Tracking in Nonminimum Phase Boost DC/DC Converter Using Sliding Mode Techniques
}

\author{
S.Baev,$\quad$ Y.Shtessel ${ }^{\dagger}$
}

\begin{abstract}
The problem of causal output tracking in the nonminimum phase boost DC/DC power converter is studied. The extended method of stable system center (ESSC) is used for generation of a bounded reference profile for the internal state on the basis of given in real time output state reference profile. Sliding mode controller $(S M C)$ is proposed to track mentioned reference profiles, while converter parameters (load resistance and voltage source impedance), which affect the internal dynamics, are identified in real time, employing the idea of sliding mode parameter observer (SMPO). A numerical simulation illustrates the efficiency of the proposed control methodology in the presence of internal uncertainties and external disturbances.
\end{abstract}

\section{Introduction}

Switched power DC/DC converters are used in a big variety of real life applications [1-3], including generation of a set of DC voltages from one DC power supply, having all the converters been linked through the impedance of the source battery $[4,5]$. Not only a constant DC voltage, but also given in real time commanded voltage profile of the preserved polarity, can be generated using mentioned power converters [4-6]. In the case of boost DC/DC converter, the nonminimum phase [7-9] nature of the latter requires special attention. In particular, direct regulating of the output voltage results in unstable growing of the phase current and finally causes damage of the converter. This issue has been extensively studied in the last decade and many control methods have been proposed, for instance, an indirect control of the output voltage in $[3,10]$, the method of stable system centre (SSC) in $[6,11]$ and the method of designing dynamic sliding surfaces in [4].

This work inherits the basic idea of [6], and addresses direct output voltage control. A nonlinear dynamical model of the converter in normal canonical form [7] is used to de-

${ }^{*} \mathrm{~S}$. Baev is with School of Computer and Information Sciences, Georgia SouthWestern State University, Americus, Georgia 31709, USA, (e-mail: sbaev@canes.gsw.edu)

$\dagger$ Y. Shtessel is with Department of Electrical and Computer Engineering, University of Alabama in Huntsville, Huntsville, Alabama 35899 USA, (email: shtessel@eng.uah.edu) sign a control law. Squared output voltage is treated as the system output, while the sum of squared input current and squared output voltage is treated as unstable internal state. A bounded reference profile for the unstable internal state is generated online using the extended method of stable system center (ESSC) [12-14].

The ESSC method allows finding a bounded particular solution of an unstable linear differential equation. Consistent use of such a method in the solution flow turns out the original output tracking problem into the state tracking one. For instance, the internal state reference profile is generated online by feeding a given output voltage command through the ESSC filter. The evaluation of such filter coefficients is based upon the proposed higher-order sliding mode (HOSM) parameter observer [15-17].

The contributions of this work can be summarized as follows:

1. Causality of the output tracking is significantly improved via allowing output reference profile being generated by unknown linear exogenous system of given order;

2. The characteristic polynomial of that exogenous system is identified online using HOSM parameter observer;

3. Internal impedance of the voltage source is included into the converter model. It limits the top level of the output voltage to be generated;

4. Load resistance and internal voltage source impedance are identified online, employing sliding mode parameter observer. The obtained estimates are used in the control law, allowing proper reaction to the change in operational conditions.

The paper is organized as follows: Section 2 is dedicated to the mathematical description of the converter, where models in natural and normalized coordinates are presented. The considered voltage tracking problem is formally stated in Section 3. Sliding mode controller is designed (Section 4) assuming availability of the internal state reference. Such an assumption is fulfilled in Section 5, where the algorithm for the internal state reference generation is presented. The design of a sliding mode parameter observer for the implicit estimation of the load resistance and the voltage source 
impedance, is covered in Section 6. A numerical simulation (Section 7) illustrates the efficiency of the proposed control methodology. The list of comprehensive conclusions (Section 8) is followed by the Appendix where the ESSC method is proven.

\section{Model of the Boost DC/DC Converter}

A boost DC/DC electric power converter (Fig. 1) can be modeled according to the following system of nonlinear differential equations:

$$
\left\{\begin{array}{l}
L \frac{d i}{d \tau}=-i r+E-V_{0} u \\
C \frac{d V_{0}}{d \tau}=-\frac{1}{R} V_{0}+i u
\end{array}\right.
$$

where $u \in[0,1]$ is the control input; $i$ is the inductor current (available for measurement); $V_{0}$ is the output voltage (available for measurement); $L$ is a known input inductance; $r$ is an impedance of the DC voltage source; $C$ is a known output capacitance; $R$ is an equivalent load resistance; $\tau$ is real time.

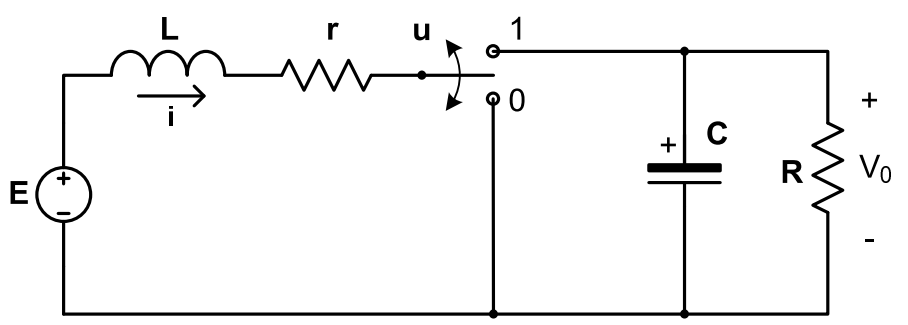

Figure 1. Boost $D C / D C$ converter circuit

The model (1) is presented in natural coordinates, where the input current and the output voltage are the model states. This particular presentation of the plant model does not make its nonminimum phase nature to show up. More convenient presentation of the converter dynamics, from the control law design standpoint, is to be used. Introduce a normalization of the model parameters along with the new state vector, which allows writing the model (1) in the normal canonical form [7]:

$$
\begin{aligned}
& \frac{d y}{d t}=\dot{y}=-\frac{2}{\gamma_{R}} y+\sqrt{y(\eta-y)}+v \sqrt{y(\eta-y)} \\
& \frac{d \eta}{d t}=\dot{\eta}=-2 \gamma_{r}(\eta-y)+2 \sqrt{\eta-y}-\frac{2}{\gamma_{R}} y
\end{aligned}
$$

where

$y=\frac{1}{E^{2}} V_{0}^{2}$ is the output state;

$\eta=\frac{1}{E^{2}} V_{0}^{2}+\frac{L}{C E^{2}} i^{2}$ is the internal state;

$v \in[-1,1]$ is the "new" control, defined as $v=2 u-1$;

$\gamma_{r}=r \sqrt{C / L}$ is the source quality factor;

$\gamma_{R}=R \sqrt{C / L}$ is the load factor;
$t=\frac{\tau}{\sqrt{L C}}$ is the "new" (scaled) model time.

System (2) is presented in a form of input/output dynamics (2a), and internal (forced zero) dynamics (2b). Due to instability of the latter, the system is of nonminimum phase.

The internal dynamics (2b) appear to be presented as highly nonlinear differential equation which does not allow to apply the proposed methodology (ESSC method). The linearization of the latter in a small vicinity of some operating point $\left\{y_{0}, \eta_{0}\right\}^{T}$ is proposed to overcome such an issue. The idea is to present the nonlinear dynamics $(2 b)$ in the linearized form

$$
\dot{\eta}=Q \eta+K y+S,
$$

where piece-wise constant coefficients $Q>0, K$, and $S$ are functions of $\gamma_{r}, \gamma_{R}, y_{0}, \eta_{0}$ and are assumed to be known.

Consider a small vicinity of the operating point, where the linearized form (3) is valid (fairly presents nonlinear internal dynamics). The pair $\left(y_{0}, \eta_{0}\right)$ is supposed to be a solution of the right-hand-side of $(2 b)$

$$
\eta_{0}=y_{0}+\left(\frac{1}{2 \gamma_{r}}-\sqrt{\frac{1}{4 \gamma_{r}^{2}}-\frac{y_{0}}{\gamma_{r} \gamma_{R}}}\right)^{2}
$$

which is real, only if the solvability condition holds

$$
\frac{1}{4 \gamma_{r}^{2}}-\frac{y_{0}}{\gamma_{r} \gamma_{R}} \geq 0 \quad \therefore \quad y_{0} \leq \frac{R}{4 r}
$$

The coefficients of the linearized internal dynamics, given by (3), can now be evaluated as follows

$$
\begin{gathered}
Q=-2 \gamma_{r}+\frac{1}{\sqrt{\eta_{0}-y_{0}}}>0, \quad S=\sqrt{\eta_{0}-y_{0}}, \\
K=2 \gamma_{r}-\frac{2}{\gamma_{R}}-\frac{1}{\sqrt{\eta_{0}-y_{0}}} .
\end{gathered}
$$

Remark 1 An uncertainty of the generally unknown parameters $\gamma_{r}$ and $\gamma_{R}$ may become a reason of significant reduction of the linearization quality since they directly effect coefficients $Q$ and $K$. This issue can be addressed by employing a sliding mode parameter observer (SMPO), presented in Section 6, which allows asymptotic reconstruction of $\gamma_{r}$ and $\gamma_{R}$.

\section{The Problem Formulation}

The problem is in designing of a control law $v(t)$, which will provide causal output tracking of a causal commanded (reference) output profile $y_{c}(t)$ :

$$
\lim _{t \rightarrow \infty}\left\|y(t)-y_{c}(t)\right\|=0
$$

in the presence of bounded uncertainties of converter parameters $\gamma_{r}$ and $\gamma_{R}$, whose nominal values $\gamma_{r_{0}}$ and $\gamma_{R_{0}}$ are assumed to be known.

Remark 2 Output reference profile $y_{c}(t)$ is assumed to be generated by unknown linear exogenous system of given order. Its characteristic polynomial is identified online and then is used in the control law design. 


\section{Sliding Mode Controller Design}

The originally introduced problem of direct output voltage tracking is reduced to the conventional state tracking. In other words, given reference profile $y_{c}$ is used twice: as a desirable trajectory for the output state $y$, and as an input of the ESSC filter which generates a bounded reference profile $\eta_{c}$ for the unstable internal state $\eta$ (see Section 5 for details on ESSC filter design).

Assume that profile $\eta_{c}$, satisfying the linear differential equation (3), is known. A sliding mode control law, forcing the asymptotic state tracking

$$
\lim _{t \rightarrow \infty}\left\|y(t)-y_{c}(t)\right\|=0, \quad \lim _{t \rightarrow \infty}\left\|\eta(t)-\eta_{c}(t)\right\|=0
$$

can now be designed. First of all, the two state tracking errors are introduced

$$
e_{y}=y_{c}-y, \quad e_{\eta}=\eta_{c}-\eta,
$$

and their dynamics are identified along the system trajectory

$$
\begin{aligned}
& \dot{e}_{y}=\dot{y}_{c}+\frac{2}{\gamma_{R}} y-\sqrt{y(\eta-y)}-v \sqrt{y(\eta-y)}, \\
& \dot{e}_{\eta}=Q e_{\eta}+K e_{y} .
\end{aligned}
$$

Introduce a sliding variable $\sigma=e_{y}+T e_{\eta}$, where the design constant $T \in \mathbb{R}$ is to be selected to provide a desirable rate of compensated error stabilization in the sliding mode.

The $\sigma$-dynamics are identified as

$$
\begin{aligned}
\dot{\sigma} & =\psi-b v, \\
\psi & =\dot{y}_{c}+\frac{2}{\gamma_{R}} y-\sqrt{y(\eta-y)}+T Q e_{\eta}+T K e_{y}, \\
b & =\sqrt{y(\eta-y)} .
\end{aligned}
$$

The traditional sliding mode control law $[1,2]$

$$
v=\operatorname{sign}(\sigma)
$$

stabilizes $\sigma$ at the origin in a finite time. In the sliding mode $(\sigma=0)$, the following dynamics of a reduced order describes the motion of the system

$$
\begin{aligned}
& \dot{e}_{\eta}=Q e_{\eta}+K e_{y}, \\
& e_{y}=-T e_{\eta} .
\end{aligned}
$$

The design constant $T$ can now be selected to provide a desirable eigenvalue placement of $(Q-K T)$, which is responsible for the compensated error stabilization rate.

Remark 3 Finite time stabilization of the sliding variable $\sigma$ guarantees, that the tracking errors (especially $e_{\eta}$ whose dynamics are purely unstable due to $Q>0$ ) will not diverge tremendously during the reaching phase. Instead, they will take some bounded values - starting point for asymptotic convergence to the origin with the selected eigenvalue $(Q-$ $K T)$.

\section{Generation of a Bounded Profile $\eta_{c}$}

It was assumed for the purpose of the controller design, that bounded profile $\eta_{c}$ satisfies the unstable differential equation (3), therefore, the generation process is equivalent to the finding of a bounded particular solution of

$$
\dot{\eta}_{c}=Q \eta_{c}+K y_{c}+S
$$

The instability of (11) does not allow direct numerical integration. Instead, the extended method of stable system center (ESSC) is to be employed to estimate/generate $\eta_{c}$.

\subsection{The Extended Method of Stable System Center}

The ESSC method allows estimating of a bounded particular solution of the unstable differential equation (11), rewritten for the convenience as follows

$$
\dot{\eta}_{c}=Q \eta_{c}+\theta, \quad \theta=K y_{c}+S
$$

where $Q>0$ and $\theta$ is a causal forcing term available for measurement.

Remark 4 The ESSC method is designed (as presented below) to handle MIMO problems where $Q \equiv \mathbf{Q} \in \mathbb{R}^{p \times p}$ is a non-Hurwitz matrix while $y_{c} \equiv \mathbf{y}_{c} \in \mathbb{R}^{p}$ and $\theta \in \mathbb{R}^{p}$ are vectors. The obvious reduction of the method to address the mentioned SISO problem $(p=1)$ is trivial and is left uncovered.

The basis for the ESSC method - the method of Stable System Center (SSC) [9], assumes that the unstable differential equation (12) is forced by a causal (available in current time only) term $\theta$, which can be piece-wise modeled by an LTI exogenous system with the known characteristic polynomial. An extension, that turns out the SSC into the ESSC, relaxes this assumption, and requires knowing only the order of the mentioned exogenous system. A corresponding characteristic polynomial is reconstructed online using a HOSM-based parameter observer [17]. This innovation significantly improves the causality of the problem. The development of the proposed ESSC method is based on the following Lemma.

Lemma 1 (Polynomial Reconstruction) Given an LTI system of known order $k$

$$
\begin{aligned}
\dot{z} & =A z \\
\theta & =C z
\end{aligned}
$$

where $z \in \mathbb{R}^{k}, \theta \in \mathbb{R}^{p}, k \geq p$ so that:

$i$. the output $\theta$ available for measurement;

ii. the unknown matrices $A \in \mathbb{R}^{k \times k}, C \in \mathbb{R}^{p \times k}$ are supposed to satisfy the observability condition:

$$
\begin{aligned}
& M=\left[C^{T}, A^{T} C^{T}, \ldots,\left(A^{k-1}\right)^{T} C^{T}\right]^{T}, \\
& \operatorname{rank}(M)=k, \quad M \in \mathbb{R}^{p k \times k} .
\end{aligned}
$$


iii. the eigenvalues of the matrix $A$ are located in the left half of the complex plane or on the imaginary axis (nonrepeated).

\section{then}

a. there exists a constant matrix $\tilde{A}$, similar to the matrix A, that satisfies an algebraic equation

$$
\gamma(t)=\tilde{A} \psi(t)
$$

where

$$
\begin{aligned}
& \psi(t)=D \omega^{1}(t), \quad \gamma(t)=D \omega^{2}(t) \\
& \omega^{1}(t)=\left[\theta^{T}, \dot{\theta}^{T}, \ldots,\left(\theta^{(k-1)}\right)^{T}\right]^{T} \in \mathbb{R}^{p k} \\
& \omega^{2}=\dot{\omega}^{1}
\end{aligned}
$$

and the vectors $\omega^{1}(t), \omega^{2}(t)$ are obtained in real time by differentiating the output $\theta(t)$ using HOSM differentiator [15]. The arbitrary, but known design constant matrix $D \in \mathbb{R}^{k \times p k}$ is assumed to be of full rank, i.e. $\operatorname{rank}(D)=k$

b. the entries of the matrix $\tilde{A}$ can be reconstructed as

$$
\tilde{A}=\frac{\int_{t-k \Delta}^{t} \operatorname{det}(\Psi(\tau)) \Gamma(\tau) \operatorname{adj}(\Psi(\tau)) d \tau}{\int_{t-k \Delta}^{t}[\operatorname{det}(\Psi(\tau))]^{2} d \tau}
$$

where

$$
\begin{aligned}
& \Psi(t)=\left[\begin{array}{llll}
\psi\left(t_{0}\right) & \psi\left(t_{1}\right) & \ldots & \psi\left(t_{k-1}\right)
\end{array}\right] \\
& \Gamma(t)=\left[\begin{array}{llll}
\gamma\left(t_{0}\right) & \gamma\left(t_{1}\right) & \ldots & \gamma\left(t_{k-1}\right)
\end{array}\right],
\end{aligned}
$$

with $t_{i}=t-i \Delta$, and $\Delta>0$ is a constant time interval.

Proof: The proof is indirectly given in $[12,13]$ and is sketched here for brevity.

1. Vector $\theta$ is differentiated $(k+1)$ times using HOSM differentiator [15].

2. Auxiliary vectors $\omega^{1}$ and $\omega^{2}$ are constructed according to (15).

3. An arbitrary constant matrix $D \in \mathbb{R}^{k \times p k}$ of full rank is introduced to provide nonsingularity of $(D M)$. Vectors $\gamma$ and $\psi$ can now be calculated according to the second part of (15).

4. Matrix $\tilde{A}$ is defined according to $\tilde{A}=(D M) A(D M)^{-1}$.

5. Considering $k$ consecutive measurements for each component of vectors $\gamma$ and $\psi$, the two auxiliary matrices $\Gamma$ and $\Psi$ can be constructed according to (17).

6. Finally, the components of matrix $\tilde{A}$ can be reconstructed according to (16) as a solution of matrix algebraical equation using the method of least square parameter estimation (LSPE).
Remark 5 Once the matrix $\tilde{A}$ estimated, it is straightforward to identify its characteristic polynomial

$$
P_{k}(\lambda)=\lambda^{k}+p_{k-1} \lambda^{k-1}+\cdots+p_{1} \lambda+p_{0},
$$

which coincides with the one for $A$ in equation (13) due to the similarity of $\tilde{A}$ and A. Eigenvalues of both matrices $A$ and $\tilde{A}$ are considered to be located in the left half of the complex plane or on the imaginary axis (non-repeated).

The remainder of the ESSC method development computing a bounded particular solution of the unstable differential equation (12), is presented in the following Theorem.

Theorem 1 (ESSC Method) Given the unstable differential equation (12), driven by a causal signal $\theta$, which is available for measurement, and the following set of conditions

$i$. the matrix $\mathbf{Q}$ in (12) is nonsingular;

ii. the internal dynamics forcing term $\theta$ can be piece-wise modeled as the output of a dynamical process given by the unknown LTI system of differential equations (13) of known order $k$

then, the estimate $\hat{\eta}_{c} \in \mathbb{R}^{p}$ for the internal state command $\eta_{c} \in \mathbb{R}^{p}$ can be generated by a matrix differential equation

$$
\begin{gathered}
\hat{\eta}_{c}^{(k)}+C_{k-1} \hat{\eta}_{c}^{(k-1)}+\ldots+C_{1} \dot{\hat{\eta}}_{c}+C_{0} \hat{\eta}_{c}= \\
-\left(\mathbf{P}_{k-1} \boldsymbol{\theta}^{(k-1)}+\ldots+\mathbf{P}_{1} \dot{\theta}+\mathbf{P}_{0} \theta\right)
\end{gathered}
$$

where the numbers $C_{0}, C_{1}, \ldots, C_{k-1}$ are chosen to provide desired eigenvalue placement of convergence $\hat{\eta}_{c} \rightarrow \eta_{c}$, and the matrices $\mathbf{P}_{k-1}, \ldots, \mathbf{P}_{1}, \mathbf{P}_{0} \in \mathbb{R}^{p \times p}$ are given by:

$$
\begin{aligned}
\mathbf{P}_{k-1} & =\left(\mathbf{I}+C_{k-1} \mathbf{Q}^{-1}+\ldots+C_{0} \mathbf{Q}^{-k}\right) \times \\
& \times\left(\mathbf{I}+p_{k-1} \mathbf{Q}^{-1}+\ldots+p_{0} \mathbf{Q}^{-k}\right)^{-1}-\mathbf{I} \\
\mathbf{P}_{k-2} & =C_{k-2} \mathbf{Q}^{-1}+\ldots+C_{0} \mathbf{Q}^{-(k-1)}-\left(\mathbf{P}_{k-1}+\mathbf{I}\right) \times \\
& \times\left(p_{k-2} \mathbf{Q}^{-1}+\ldots+p_{0} \mathbf{Q}^{-(k-1)}\right) \\
& \vdots \\
\mathbf{P}_{1} & =C_{1} \mathbf{Q}^{-1}+C_{0} \mathbf{Q}^{-2}-\left(\mathbf{P}_{k-1}+\mathbf{I}\right) \times \\
& \times\left(p_{1} \mathbf{Q}^{-1}+p_{0} \mathbf{Q}^{-2}\right) \\
\mathbf{P}_{0} & =C_{0} \mathbf{Q}^{-1}-\left(\mathbf{P}_{k-1}+\mathbf{I}\right) p_{0} \mathbf{Q}^{-1}
\end{aligned}
$$

where the coefficients $p_{0}, p_{1}, \ldots, p_{k-1}$ of the characteristic polynomial (18) are reconstructed based on Lemma 1.

Proof: The proof is given in [9]. 


\section{Sliding Mode Parameter Observer}

The sliding mode parameter obserever is used to estimate values of uncertain parameters $\gamma_{r}$ and $\gamma_{R}$. Such values are then used for evaluation of the linearized internal dynamics' coefficients $Q$ and $K$ as (6) in (3). The "hat" notation is introduced here to differentiate the estimates and the real (existing) values, e.g. $\hat{\gamma}_{r}$ stands for the estimate of $\gamma_{r}$. That "hat"-noted estimations are to be used in (6) and thereafter instead of $\gamma_{r}$ and $\gamma_{R}$ respectively, making all following derivations dependant on the output of SMPO.

Consider the original nonlinear dynamics of the converter given in (2), where uncertain parameters $\gamma_{r}$ and $\gamma_{R}$ are assumed to be piece-wise constants with known nominal values $\gamma_{r_{0}}$ and $\gamma_{R_{0}}$ respectively.

Introduce a sliding mode observer dynamics:

$$
\left\{\begin{array}{l}
\dot{\hat{y}}=-\frac{2}{\gamma_{R_{0}}} \hat{y}+\sqrt{y(\eta-y)}+v \sqrt{y(\eta-y)}+\mu_{1} \\
\dot{\hat{\eta}}=-2 \gamma_{r_{0}}(\hat{\eta}-\hat{y})+2 \sqrt{\eta-y}-\frac{2}{\gamma_{R_{0}}} \hat{y}+\mu_{2}
\end{array}\right.
$$

where $\hat{y}, \hat{\eta}$ are the observer states, and $\mu=\left\{\mu_{1}, \mu_{2}\right\}^{T}$ is a vector injection term to be designed. Introduce an observation error:

$$
\hat{\mathbf{e}}=\left\{\hat{e}_{y}, \hat{e}_{\eta}\right\}^{T}, \quad \hat{e}_{y}=y-\hat{y}, \quad \hat{e}_{\eta}=\eta-\hat{\eta}
$$

which dynamics are identified as follows

$$
\dot{\hat{\mathbf{e}}}=\left[\begin{array}{cc}
\hat{y} & 0 \\
\hat{y} & \hat{\eta}-\hat{y}
\end{array}\right]\left[\begin{array}{c}
2 / \gamma_{R_{0}} \\
2 \gamma_{r_{0}}
\end{array}\right]-\left[\begin{array}{cc}
y & 0 \\
y & \eta-y
\end{array}\right]\left[\begin{array}{c}
2 / \gamma_{R} \\
2 \gamma_{r}
\end{array}\right]-\mu
$$

The injection term $\mu$ is proposed to be designed according to unit vector control (UVC) approach [1,2]:

$$
\mu=\rho \frac{\hat{\mathbf{e}}}{\|\hat{\mathbf{e}}\|}
$$

where design constant $\rho>0$ should be selected big enough to provide a finite time stabilization of the observation error e.

In the sliding mode $(\mathbf{e}=\mathbf{0})$, which is established by injection (24) in finite time, the following equality holds:

$$
\left[\begin{array}{cc}
\hat{y} & 0 \\
\hat{y} & \hat{\eta}-\hat{y}
\end{array}\right]\left[\begin{array}{c}
2 / \gamma_{R_{0}}-2 / \gamma_{R} \\
2 \gamma_{r_{0}}-2 \gamma_{r}
\end{array}\right]=\mu_{e q}
$$

where $\mu_{e q}$ is the equivalent injection that can be asymptotically reconstructed by entry-wise low-pass filtering (LPF) of the discontinuous vector term $\mu$ :

$$
\hat{\mu}_{e q_{j}}=\operatorname{LPF}\left(\mu_{j}\right), \quad j=1,2
$$

Finally, estimates $\hat{\gamma}_{r}$ and $\hat{\gamma}_{R}$ can be reconstructed from the solution of linear system (25):

$$
\begin{gathered}
\hat{\gamma}_{R}=\left(\frac{1}{\gamma_{R_{0}}}-\frac{z_{1}}{2}\right)^{-1}, \quad \hat{\gamma}_{r}=\gamma_{r_{0}}-\frac{z_{2}}{2}, \\
{\left[\begin{array}{l}
z_{1} \\
z_{2}
\end{array}\right]=\left[\begin{array}{cc}
\hat{y} & 0 \\
\hat{y} & \hat{\eta}-\hat{y}
\end{array}\right]^{-1} \hat{\mu}_{e q} .}
\end{gathered}
$$

\section{Numerical Simulations}

A numerical simulation of the boost DC/DC converter performance has been accomplished using VisSim ${ }^{1}$ simulation software.

Boost converter model in the normal canonical form (2) has been used for simulation purposes. Reference profile $y_{c}$ is assumed to be constructed as sinusoidal signal of arbitrary piece-wise magnitude and frequency, shifted by arbitrary positive piece-wise constant DC level. Such level is estimated by feeding signal $y_{c}$ through a low-pass filter, and is used to calculate the operating point component $y_{0}$. The other component $\eta_{0}$ is calculated according to (4).

\subsection{Simulation details and plots}

A $3^{\text {rd }}$ order linear exogenous system can be used to describe the dynamics of $y_{c}$ profile, which yields the same $3^{\text {rd }}$ order of the ESSC filter.

For simulation purposes, output reference profile $y_{c}$ defined as follows:

$$
y_{c}(t)= \begin{cases}5+0.5 \sin (3 t), & t \leq 20 \\ 10, & 20<t \leq 60 \\ 10+\sin (1.5 t), & 60<t \leq 75 \\ 15+1.5 \sin (5 t), & t>75\end{cases}
$$

Piece-wise constant uncertain parameters $\gamma_{r}$ and $\gamma_{R}$ are defined as:

$$
\gamma_{R}=\left\{\begin{array}{ll}
2.53, & t \leq 40 \\
3.79, & t>40
\end{array}, \quad \gamma_{r}= \begin{cases}3.79 \times 10^{-3}, & t \leq 40 \\
2.53 \times 10^{-3}, & t>40\end{cases}\right.
$$

while the nominal values are known as $\gamma_{R_{0}}=3.16$ and $\gamma_{r_{0}}=$ $3.16 \times 10^{-3}$ respectively.

Simulation plots are shown in Figs. 2-3.

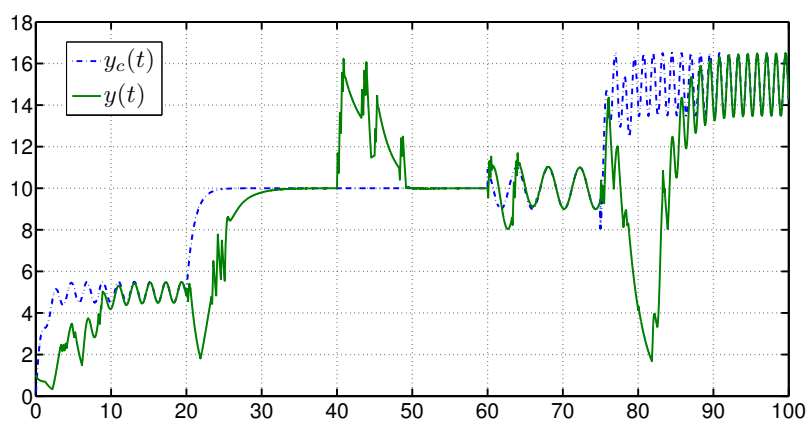

Figure 2. Output state tracking performance

The two plots illustrate the output and internal state tracking performance. There are number of brakes during the tracking process, caused by abrupt change of either circuit parameters or tracking profile dynamics. Each break is

\footnotetext{
${ }^{1}$ VisSim User's Guide, Version 7.0, Copyright 1990-2007 Visual Solutions, Inc., 487 Groton Road, Westford, MA, 01886
} 


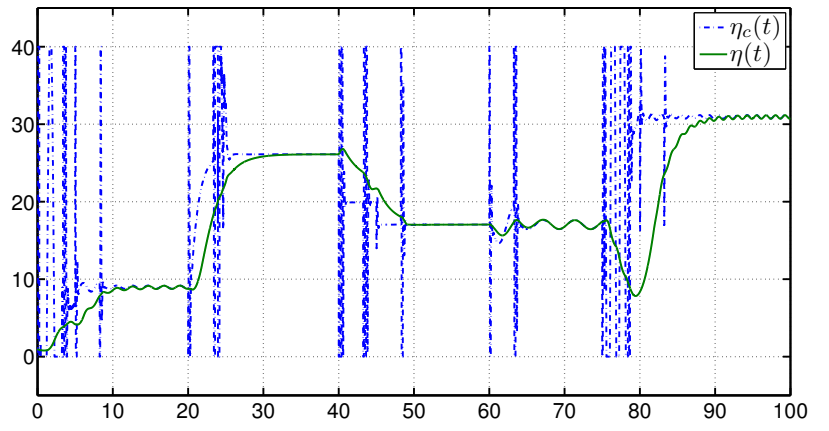

Figure 3. Internal state tracking performance

detected as a destruction of the sliding mode, and is followed by the reconstruction phase, where all major coefficients are recalculated and are used for further tracking.

\subsection{Discussion}

The output reference profile $y_{c}$ is selected to have both $\mathrm{DC}$ and $\mathrm{AC}$ components, but having the preserved polarity. This allows authors to illustrate the efficacy of the technique, i.e. tracking of a causal output profile without breaking the main condition of preserving DC character of the output signal.

\section{Conclusions}

The problem of causal output tracking for the nonminimum phase boost DC/DC converter has been studied.

1. The direct causal output voltage tracking, i.e. $y \rightarrow y_{c}$ as time increases, is accomplished for the reference profile $y_{c}$, given in real time, in the presence of uncertain impedance of the voltage source and equivalent resistance of the load.

2. The solvability condition (5), which limits the highest DC level of the converter output voltage, is derived.

3. Bounded reference profile for the unstable internal state is generated online, employing the extended method of stable system center.

4. The sliding mode control law is designed to asymptotically stabilize the state tracking errors in the presence of unmatched by control uncertain parameters.

5. Sliding mode parameter observer is designed to estimate uncertain impedance of the voltage source and equivalent resistance of the load. Obtained estimates are used in the control law.

6. Numerical simulation confirms the efficiency of the proposed control methodology.

\section{References}

[1] V. Utkin, J. Gulder, and M. Shijun, Sliding Mode Control in Electromechanical Systems. Taylor \& Francis, 2nd ed., 1999.
[2] C. Edwards and S. Spurgeon, Sliding Mode Control. Bristol, PA: Taylor \& Francis, 1998.

[3] H. Sira-Ramirez and M. Rios-Bolivar, "Sliding mode control of DC/DC power converters via extended linearization," IEEE Transactions on Circuits and Systems, vol. 41, no. 10, pp. 652-661, 1994.

[4] Y. Shtessel, O. Raznopolov, and L. Ozerov, "Control of multiple modular DC/DC power converters in conventional and dynamic sliding surfaces," IEEE Transactions on Circuits and Systems, vol. 45, no. 10, pp. 1091-1101, 1998.

[5] A. Zinober, Y. Shtessel, E. Fossas, J. Olm, and B. Patterson, "Nonminimum phase output tracking control strategies for DC/DC power converters," in Lecture Notes in Control and Information Sciences (E. Fossas, C. Edwards, and L. Fridman, eds.), pp. 447-482, Berlin: Springer-Verlag, June 2006.

[6] Y. Shtessel, A. Zinober, and I. Shkolnikov, "Sliding mode control of boost and buck-boost power converters using method of stable system centre," Automatica, vol. 39, no. 6, pp. 1061-1067, 2003.

[7] A. Isidori and C. Byrnes, "Output regulation of nonlinear systems," IEEE Transactions on Automatic Control, vol. 35, pp. 131-140, 1990.

[8] S. Gopalswamy and J. Hedrick, "Tracking nonlinear nonminimum phase systems using sliding control," International Journal of Control, vol. 57, pp. 1141-1158, 1993.

[9] I. Shkolnikov and Y. Shtessel, "Tracking in a class of nonminimum-phase systems with nonlinear internal dynamics via sliding mode control using method of system center," Automatica, vol. 38, pp. 837-842, 2002.

[10] E. Fossas and J. Olm, "Asymptotic tracking in DC/DC nonlinear power converters," Discrete and Continuous Dynamical Systems, Series B, vol. 2, no. 2, pp. 295-307, 2002.

[11] Y. Shtessel, A. Zinober, and I. Shkolnikov, "Sliding mode control for nonlinear systems with output delay via method of stable system centre," ASME Journal of Dynamic Systems, Measurement and Control, vol. 25, no. 2, pp. 253-257, 2003.

[12] S. Baev, Y. Shtessel, and I. Shkolnikov, "Nonminimum-phase output tracking in causal systems using higher order sliding modes," International Journal of Robust and Nonlinear Control, vol. 18, no. 4-5, pp. 454-467, 2008. Special Issue on Advances in Higher Order Sliding Mode Control.

[13] S. Baev, Y. Shtessel, and C. Edwards, "HOSM observer for a class of non-minimum phase causal nonlinear MIMO systems," in Proceedings of 17th IFAC World Congress, (Seoul, Korea), 2008.

[14] S. Baev, Y. Shtessel, and I. Shkolnikov, "HOSM driven output tracking in the nonminimum-phase causal nonlinear systems," in Proceedings of CDC'07, (New Orleans, LA, USA), 2007.

[15] A. Levant, "Higher order sliding modes, differentiation and output feedback control," International Journal of Control, vol. 26, no. 9, pp. 924-942, 2003.

[16] Y. Shtessel and A. Poznyak, "Parameter identification of affine time varying systems using traditional and high order sliding modes," in Proceedings of ACC'05, (Portland, Oregon, USA), 2005.

[17] S. Baev, I. Shkolnikov, Y. Shtessel, and A. Poznyak, "Sliding mode parameter identification of dynamic systems with measurement noise," International Journal of Systems Science, vol. 38, no. 11, pp. 871-878, 2007. Special Issue on Advances in Sliding Mode Observation and Estimation (part two). 